\title{
Purely Dynamical Signature of the Orientational Glass Transition
}

\author{
M. Jiménez-Ruiz, ${ }^{1}$ A. Criado, ${ }^{1}$ F. J. Bermejo, ${ }^{1}$ G. J. Cuello, ${ }^{1}$ F. R. Trouw,${ }^{2}$ R. Fernández-Perea, ${ }^{2}$ H. Löwen, ${ }^{3}$ \\ C. Cabrillo, ${ }^{1}$ and H. E. Fischer ${ }^{4}$ \\ ${ }^{1}$ Consejo Superior de Investigaciones Cientficas, Serrano 121-123, E-28006 Madrid, Spain \\ and Departamento de Física de la Materia Condensada, Universidad de Sevilla, P.O. Box 1065, E-41080 Seville, Spain \\ ${ }^{2}$ Argonne National Laboratory, Argonne, Illinois 60439 \\ ${ }^{3}$ Institut für Theoretische Physik II, Heinrich-Heine-Universität, Universitätsstrasse 1, D-40225 Düsseldorf, Germany \\ ${ }^{4}$ Institut Laue Langevin, BP 156x, F-38042 Grenoble Cedex 9, France
}

(Received 16 March 1999)

\begin{abstract}
The dynamics of the freezing transition of the rotator phase crystal of ethanol into its orientational glass phase is monitored by measurements of molecular rotational components in the quasielastic neutron scattering spectrum. We demonstrate that phenomena observed at pico- and nanosecond scales can be mapped onto those shown by a model of infinitely thin hard needles rotating around bodycentered-cubic lattice positions. As the model glass transition is of purely dynamical origin, our findings support the idea that the glass transition is purely dynamical and not associated with any thermodynamic phase transition.

PACS numbers: 64.70.Pf, 61.20.Lc, 61.43.-j
\end{abstract}

The nature of the nonequilibrium transition which occurs by rapid cooling of a liquid, the canonical liquid-glass transition, remains an elusive question [1]. In a nutshell, the transition may be viewed either as a purely dynamical phenomenon without any associated changes in static quantities [2] or as a remnant of an underlying thermodynamic phase change $[3,4]$ which is partially hidden by kinetic reasons. Arguments in favor of both points of view are partially supported by experimental data. On the other hand, both alternatives are grounded on fairly well elaborated theoretical frameworks such as kinetic theories of the mode-coupling family [2] and those of the dynamics of critical phenomena [3].

The main difficulties in evaluating the merits of both approaches concern the wide range of complex phenomena involved within the glass transition of a real material which hides some of the behaviors expected to appear as characteristic signatures of the transition. In fact, most systems where detailed studies can be carried out within the deeply supercooled liquid do show a rich variety of phenomena such as molecular rotations and/or low-energy vibrations, which are strongly coupled to the translational dynamics due to the huge viscosity characteristic of temperatures near the glass-transition point $T_{\mathrm{g}}$. While some progress on the understanding of the effect of such motions on quantities usually employed to study the dynamic correlations at temperatures close to $T_{\mathrm{g}}$ has been achieved [5], the question remains as to whether the structural liquid-glass transition is purely dynamical in origin.

The aim of this Letter is to test whether the glass transition leading from rotator phase crystals (RP) to the orientationally disordered [orientational glass (OG)] state can be understood as a purely dynamical crossover. In order to do so, we compare neutron scattering data for ethanol across the above mentioned transition with that resulting from a simple, albeit nontrivial model [6] which exhibits a purely dynamical crossover. We find very similar signatures of the RP $\rightarrow$ OG transition in both cases which supports the idea that such a transition is a purely dynamic phenomenon.

Our motivation to monitor the RP $\rightarrow$ OG transition on ethanol is threefold: First, while the freezing of RP crystals has been studied in a fair number of systems [7], many details of the RP $\rightarrow$ OG transition have been clarified recently for ethanol $[8,9]$. Second, the presence of longrange positional periodicity at both sides of the RP $\rightarrow$ OG transition implies that the melting process is purely rotational (rotation-translation coupling effects are reasonably small [10]), and can thus be followed by monitoring the neutron quasielastic scattering across the transition, in contrast with the canonical glass transition where the rotational melting is partially hidden by the emergence of quasielastic intensities from other sources. Third, ethyl alcohol has the unique feature that it can be prepared in two different phases showing glassy behavior at the same temperature, one of these phases having structural disorder (liquid and glass) and the other having only orientational disorder (RP and $\mathrm{OG}$ ). In the disordered crystals the molecular centers of mass sit at the nodes of a bcc lattice $[10,11]$, and melting into the RP is signaled by jumps in specific heat [9] and thermal expansivity. The close proximity of both transitions was also revealed by dielectric spectroscopy [12] where both $\alpha-$ and sub $-T_{\mathrm{g}}$ relaxations appear astoundingly near in frequency and temperature dependence. Hence the canonical glass transition seems to be dominated by the freezing of the orientational degrees of freedom. If there is evidence that the $\mathrm{RP} \rightarrow \mathrm{OG}$ is purely dynamic, the same conclusion should apply for the canonical glass transition. Hence our study even sheds new light on the nature of the structural liquid-glass transition.

Two sets of neutron scattering experiments were carried out. Exploration of the microscopic dynamics within a 
scale of about $1 \mathrm{meV}\left(\approx 1.5 \mathrm{ps}^{-1}\right)$ was pursued using the inverted-geometry time-of-flight spectrometer QENS at the Intense Pulsed Neutron Source, whereas that taking place at $\mu \mathrm{eV}$ scales was monitored using the IN16 backscattering spectrometer at the Institut Laue Langevin (Grenoble). The former instrument operates with a fixed final energy of $3.65 \mathrm{meV}$ enabling an energy resolution of $\Delta E \sim 90 \mu \mathrm{eV}$ (HWHM), whereas the latter was set up within a configuration which delivered a resolution of $1 \mu \mathrm{eV}$ and an energy-transfer range up to $\pm 15 \mu \mathrm{eV}$. Preparation of glass and RP crystal samples followed routes described previously $[8,11,12]$. Both liquid $\rightarrow$ glass and the $\mathrm{RP} \rightarrow \mathrm{OG}$ transitions occur in a temperature interval centered at about $97 \mathrm{~K}$. Partially $\left(\mathrm{C}_{2} \mathrm{D}_{5} \mathrm{OH}\right)$ deuterated samples were employed to monitor its state (glass, liquid, or cubic crystal) by inspection of the diffraction patterns.

The shape of all measured spectra conforms to that shown in Fig. 1. Both at meV and $\mu \mathrm{eV}$ scales it shows a strong elastic (resolution-limited) component plus a quasielastic contribution which can be seen by the naked eye. A sample of the temperature dependence of the quasielastic linewidths for two representative values of the momentum transfer $Q$ is shown in Fig. 2. No significant dependences were found for other explored $Q$ values. Apart from details arising from the rather different frequency windows and scattering power of the two spectrometers, the same dependence is observed, showing that the broadening of the quasielastic spectrum increases through the rotational melting transition.

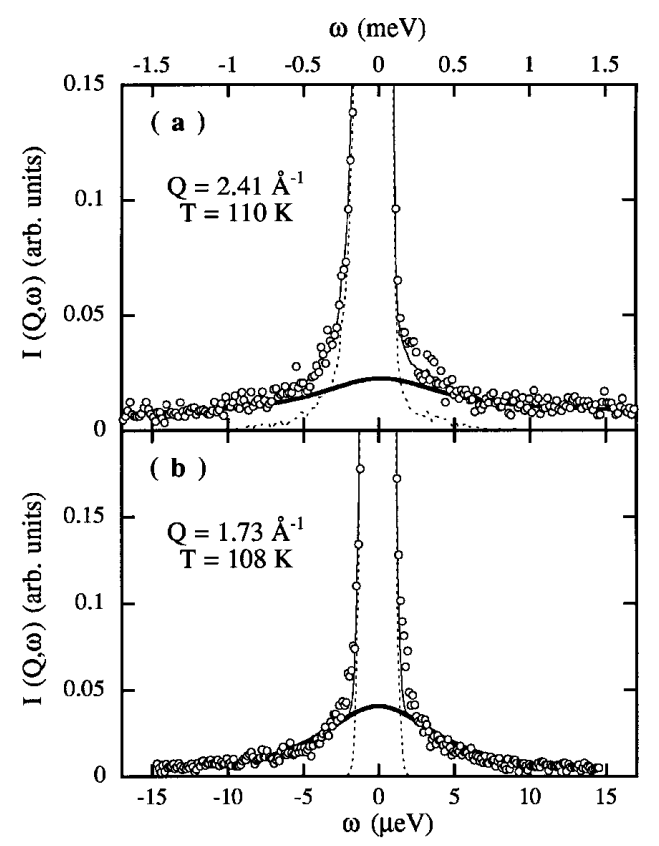

FIG. 1. Spectra as measured on both QENS (a) and IN16 (b) spectrometers. Model fits are shown by thin solid lines. Dashed and thick solid lines correspond to the elastic and quasielastic components, respectively.
The assignment of the observed broadenings to underlying microscopic motions requires the use of a model to represent the dynamics [13]. On symmetry grounds, one expects that its basic features are encompassed within the formalism describing molecular reorientations about [100], [110], and [111] axes of a cubic lattice [14]. It predicts a neutron scattering law given in terms of four classes of molecular rotations with jump rates $\tau_{j}^{-1}, j=$ 1-4. The time scales of two of these rates are set from the spectral linewidths measured with both instruments. However, their assignment to specific motions requires additional information from some other sources. To such an effect, we have carried out molecular dynamics simulations on the $N-P-T$ ensemble (the cell shape is allowed to fluctuate) using a realistic model potential for the microscopic interactions [15]. The results of such an endeavor were gratifying since (a) the equilibrium lattice structure is reproduced, (b) freezing of rotational motions occurs below $100 \mathrm{~K}$, and (c) the crystal structure becomes unstable above $120 \mathrm{~K}$ that is quite close to experiment. From the analysis of the computed trajectories, it was found that molecules will reorient between some 24 preferred orientations with vastly different rates. Reorientations leaving unaffected the most preferred orientations $(\mathrm{C}-\mathrm{O}$ bond along the [001] direction and $\mathrm{C}-\mathrm{C}$ bond close to $[11 \overline{1}]$, and those related by symmetry) were found to take place within the picosecond scale, whereas far more infrequent jumps occurring within scales of hundreds of picoseconds were also monitored. The calculated intermediate dynamic structure factor $F_{\mathrm{MD}}(Q, t)$ showed

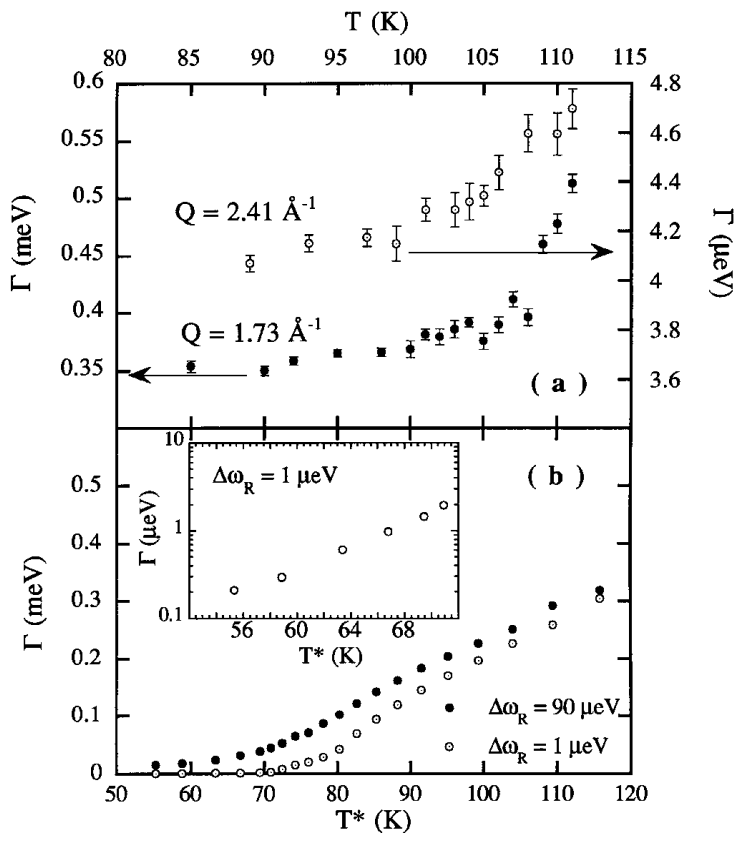

FIG. 2. (a) Temperature dependence of the quasielastic linewidth as measured on QENS (filled) and IN16 (open symbols) spectrometers. (b) Variation of the width of $\Phi(\omega)$ as calculated from the results for the system of hard needles. The inset shows data in the $\mu \mathrm{eV}$ scale on a semilogarithmic plot. 
strong deviations from exponential behavior and, in fact, four different exponential decays were needed to fully account for their shapes. The jump rates $\tau_{j}^{-1}$ estimated in such a way were found to be well separated in time. Two of those decays were found to be within the frequency windows covered by the experiments and therefore we identify the observed relaxations with motions of the two classes described above.

Apart from the variation with temperature of the linewidth, the RP $\rightarrow$ OG transition is also followed by the raise with temperature of the quasielastic intensity and the concomitant decrease of the elastic peak, as shown in Fig. 3. As seen there, the transition is very nicely monitored following the transfer of spectral intensity from elastic to quasi- (or inelastic) scattering and this shows very similar characteristics for both frequency windows.

From data given above it is seen that molecular rotations occur in the rotator-phase crystal on pico- and nanosecond scales at temperatures where the main $\alpha$ relaxation explored by dielectric spectroscopy [12] already reaches macroscopic relaxation times. Well below $80 \mathrm{~K}$ rotational freezing seems complete, and all the molecular degrees of freedom which are thermally excited will contribute to the spectrum as inelastic (i.e., finite-frequency) signals [9]. This is in very good agreement with specific heat data [9] which also show that the extent in temperatures of the transition is quite comparable to that evidenced by the present data (i.e., about $18 \mathrm{~K}$ ).

In the quest for physically simple models which exhibit a purely dynamical glass transition, a model of hard infinitely thin needles on a lattice as developed by Renner et al. [6] seems most appealing. The system is constituted by a set of hard needles that are executing free rotations between elastic collisions having their centers of mass located at the nodes of a cubic lattice. The only control parameter is the ratio $\ell=L / a$ of the needle length $L$ to the lattice constant $a$ [16]. Since the excluded volume of the needles is zero, all the static properties of the model are trivial (i.e., there are no static correlations). However, its transport and dynamical properties exhibit a strong dependence on $\ell$.

The dynamics of the needle model is investigated by computer simulations. We consider a system of $N=432$ infinitely thin needles with a homogeneous line mass density $m / L$ whose center-of-mass coordinates are fixed onto a bcc lattice in a periodically repeated cubic simulation box. We took a bcc crystal in order to incorporate the ethanol lattice structure in the RP. Calculating the trajectories of the needles we obtained the time-autocorrelation function for the needle orientations defined as $\Phi_{1}(t)=$ $\left\langle\frac{1}{N} \sum_{i=1}^{N} \vec{u}_{i}(0) \cdot \vec{u}_{i}(t)\right\rangle$, where the angular brackets denote the canonical average and $\vec{u}_{i}(t)$ is the time-dependent trajectory of the unit vector describing the orientation of the $i$ th needle. We explored the range $1.0 \leq \ell \leq 5.0$. The system was left to evolve over a long time corresponding to $10^{6}$ collisions. As a result, as $\ell$ increases the relax-

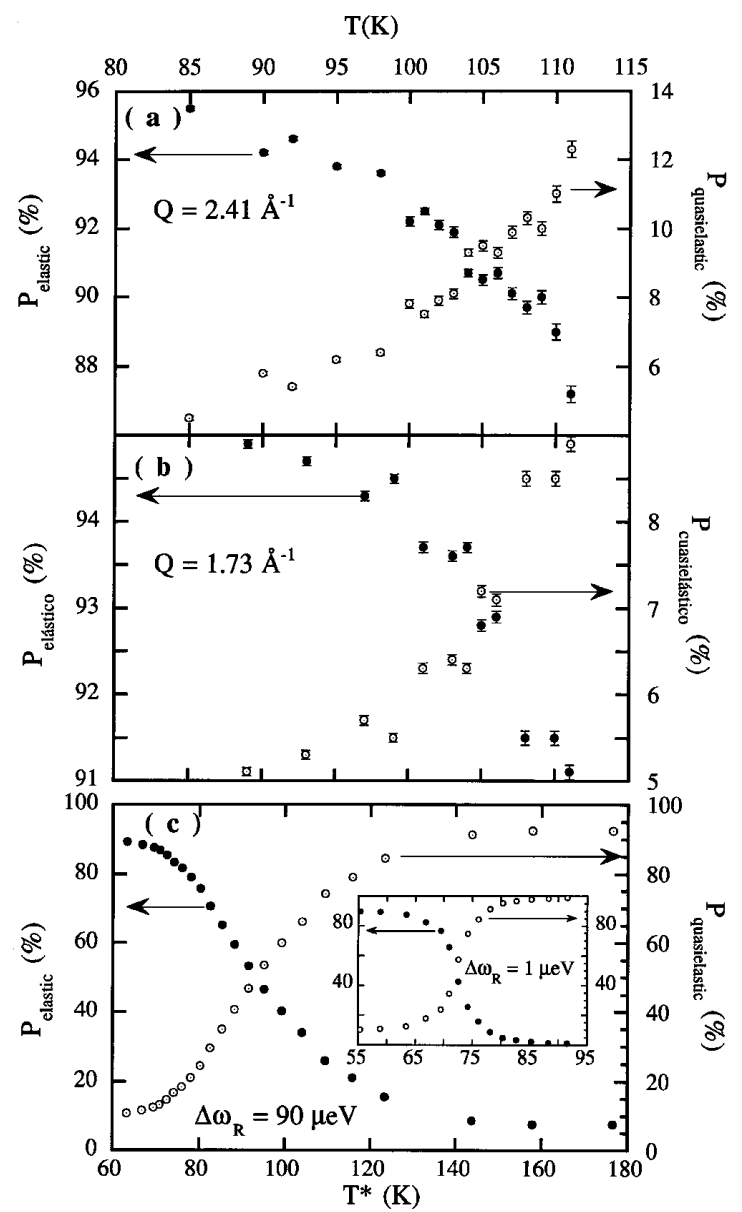

FIG. 3. (a) Temperature dependence of the elastic (solid) and quasielastic (open symbols) intensities across the RP $\rightarrow$ OG transition measured on the QENS spectrometer. (b) Same data measured on IN16. (c) Contributions from the $\Phi(\omega)$ to the elastic (solid) and quasielastic (open symbols) frequency windows versus the equivalent temperature $T^{*}$ for the resolution width $\Delta \omega_{R}=90 \mu \mathrm{eV}$. The inset shows data for the resolution width $\Delta \omega_{R}=1 \mu \mathrm{eV}$.

ation of $\Phi_{1}(t)$ becomes more and more sluggish, and for $\ell \sim 3.4$ the autocorrelation is almost blocked on the time scale explored in the simulation. For $\ell=4.5$ the orientational autocorrelation is almost equal to unity, that is orientations becoming frozen within a very narrow solid angle. Let us now discuss how to establish a link between the needle model and our experimental data. The time scale can be mapped directly. In the needle model it is set by the time $\tau \equiv \sqrt{m L^{2} / 24 k_{B} T}$. If we identify the moment of inertia of the needles $J=m L^{2} / 12$ with that of one ethanol molecule $\left(J=0.741 \times 10^{-45} \mathrm{Kg} \mathrm{m}^{2}\right)$, we obtain the fact that the time scale $\tau$ is of the order of $1 \mathrm{ps}$ at $T=100 \mathrm{~K}$ which sets the time scale. Consequently we can translate experimental frequencies into simulation data and vice versa. Finally, in the athermal needle model, only $\ell$ enters, whereas temperature is the crucial parameter for our measurements. In order to establish a mapping between $\ell$ and $T$, we write an inverse effective 
ratio $1 / \ell$ as a Boltzmann factor $1 / \ell=A \exp \left(-E / k_{B} T\right)$ [17]. Clearly the limit $\ell \rightarrow \infty$ corresponds to zero temperature. The two free constants, namely, the amplitude $A$ and the energy scale $E$, are determined as follows: First we recall that if the needle length is smaller than the nearest neighbor distance $\sqrt{3} a / 2$ the needles are noninteracting free rotators, which corresponds to infinite temperature and fixes the amplitude $A=2 / \sqrt{3}=1 / \ell_{0}$. Second, the activation energy $E$ should correspond to the experimental glass transition temperature such that $E=k_{B} T_{g}$. Hence, the translation of temperatures in the experiment into effective ratios $\ell$ of our model is given by $\ell=\ell_{0} \exp \left(T_{g} / T^{*}\right)$.

To compare experiment and model results the Fourier transform of $\Phi_{1}(t)$ into the frequency domain was evaluated. Our simulation data of $\Phi_{1}(t)$ were convoluted with the instrumental resolution functions in order to mimic the measurements. The resulting function $\Phi(\omega)$ was split into elastic and quasielastic parts depending upon the width $\Delta \omega_{R}$. The width and the amplitude of the quasielastic part were determined afterwards. The results for the widths are given in Fig. 2 and those for the intensity ratios in Fig. 3. Note that the simulation data were always expressed in temperature via the translation given above. One clearly sees a kink in the Lorentzian intensity both in the experimental data and in the transformed needle model data at temperatures about 97 and $75 \mathrm{~K}$, respectively. This is a clearcut fingerprint of the orientational glass transition. On the other hand, the elastic intensity also exhibits a similar kink. The differences between experiment and model concern the relative magnitudes of changes in elastic and quasielastic intensities as well as in the absolute values of the linewidths. Whereas experiment contains a strong elastic scattering component arising from the presence of a well-defined crystal structure having translational degrees of freedom, such a contribution is obviously absent in the model which, by construction, shows no strictly elastic component. The relative width of the crossovers are about $30 \mathrm{~K}$ in experiment and $\approx 70 \mathrm{~K}$ for the needle model, a difference expected from the absence in the latter case of a true interaction potential. The effective widths of the quasielastic spectra of both model and experiment shown in Fig. 2 exhibit crossovers at the same temperatures as do the kinks in the intensities of Fig. 3. This gives compelling evidence that the essential signatures of the orientational glass transition can be understood from a purely dynamical model.

In conclusion, we have shown that the essential features of the neutron scattering data across the orientational glass transition can be understood in terms of a purely dynamical model. The fingerprint of the transition as revealed by a cusp in the inelastic scattering is very similar in the experiment and in the model. The implications of such an analogy in dynamical behavior can, in the light of previous data, to a large extent be applied to the canonical glass-liquid transition inasmuch as the latter must carry a large rotational component (in fact, the jump in specific heat at the glass and $\mathrm{OG} \rightarrow \mathrm{RP}$ transitions corresponds to an activation of $\approx 2.8$ degrees of freedom in the latter and about 3.7 in the former, the extra degree surely assignable to translational motions). In consequence, the scenario of a transition of purely dynamical origin accounts for most of the observed signatures of the glass transition which, put into real numbers, amounts to a difference of about $20 \%$ of the jump in specific heat, an even smaller difference in the low-frequency spectra and lowtemperature properties, and a close proximity in the case of macroscopic relaxations.

This work was supported in part by the U.S. Department of Energy, Basic Energy Sciences-Materials Sciences, under Contract No. W-31-109-ENG-38 and DGICYT (Spain) Grant No. PB95-0075-C03-01.

[1] I. Gutzow and J. Schmelzer, The Vitreous State (SpringerVerlag, Berlin, 1995), p. 287.

[2] W. Götze, in Liquids, Freezing and Glass Transition, edited by J.P. Hansen et al. (North Holland, Amsterdam, 1991).

[3] J. P. Sethna et al., Phys. Rev. B 44, 4943 (1991).

[4] J. Bletry, Z. Naturforsch. A 51, 87 (1996).

[5] R. Schilling et al., Phys. Rev. E 56, 2932 (1997).

[6] C. Renner et al., Phys. Rev. E 52, 5091 (1995); S. Obukov et al. J. Phys. I (France) 7, 563 (1997).

[7] M. Descamps et al., in Quasielastic Neutron Scattering, edited by J. Colmenero et al. (World Scientific, Singapore, 1994), p. 107.

[8] M. A. Ramos et al., Phys. Rev. Lett. 78, 82 (1997).

[9] C. Talón et al., Phys. Rev. B 58, 745 (1998).

[10] F. J. Bermejo et al., Phys. Rev. B 56, 11536 (1997).

[11] R. Fayos et al., Phys. Rev. Lett. 77, 3823 (1996).

[12] M. Miller et al., Phys. Rev. B 57, R13977 (1998); M. Jiménez-Ruiz et al., Phys. Rev. B 59, 9155 (1999).

[13] S. W. Lovesey, Theory on Neutron Scattering from Condensed Matter (Oxford Science Publications, New York, 1984), Vol. I, p. 244.

[14] M. Bée, Quasielastic Neutron Scattering (Adam Hilger, Bristol, 1988), p. 228.

[15] A. Criado et al. (to be published).

[16] D. Frenkel and J. F. Maguire, Phys. Rev. Lett. 47, 1025 (1981); Mol. Phys. 49, 503 (1983).

[17] A more elaborate mapping is known for hard spheres where the effective diameter is also taken as an averaged Boltzmann factor in order to match the second virial coefficient, see, e.g., J. A. Barker et al., Rev. Mod. Phys. 48, 587 (1976). 